\title{
Am Beginn einer neuen Epoche - Verbinden und Differenzieren
}

\author{
Harald Walach \\ School of Social Sciences \& Samueli Institute for Information Biology, University of Northampton, UK
}

Wissenschaft folgt nicht festen Regeln wie Auto fahren, wo man nur die allgemeine Straßenverkehrsordnung befolgen und möglichst nüchtern und wach bleiben muss, damit man irgendwie ans Ziel kommt. Sie funktioniert auch nicht wie Klempnerei, wo man lediglich das rechte Material dabei haben muss, um mit ein bisschen Geschick und Ausbildung alles richtig zu machen. Und komplementärmedizinische Forschung ist auch nicht wie englische Klempnerei, also nur etwas schlampiger als normale, so dass sie nur richtig organisiert gehörte und alles wäre in Ordnung. Nein, Wissenschaft ist ein lebendiger, organismischer Prozess. Das Subjekt der Wissenschaft sind all jene, die Forschung betreiben und Wissen publizieren. Aber was ist das Objekt? Naiverweise würde man antworten: die Natur, das, was uns gegeben ist. Aber so einfach ist es nicht. Die Natur ist etwas, was wir Menschen dauernd verändern, herstellen, ja uns selber erst zurichten, damit wir es als Natur verstehen. Kultur - die geschaffene Wirklichkeit und Natur - die vorgefundene Wirklichkeit - sind nicht voneinander zu trennen. Kultur früherer Generationen wird Natur für die künftigen, die daraus wieder ihre eigene Kultur schaffen. So schafft sich auch Wissenschaft immer wieder selber die Wirklichkeit, die sie beforscht. Sie hat nicht nur eine abbildende Funktion, sondern ist eminent schöpferisch.

Aus diesem Grund ist es auch sträflich naiv zu glauben, man müsse nur ein guter, gesetzestreuer Fahrer im wissenschaftlichen Straßenverkehr sein, und schon ist man ein Mitspieler. Wissenschaft ist eher wie ein Straßenverkehr, in dem sich Verkehrsmittel, Verkehrsregeln, Ziel und Stil der Bewegung während des Fahrens fortlaufend ändern. Oder, um ein etwas organischeres Bild zu verwenden, Wissenschaft ist wie ein Tanz, bei dem die Tanzenden durch ihre Bewegung die Musik, nach der sie tanzen, mitgestalten, verändern, zu neuen Rhythmen und Melodien inspirieren und damit gleichzeitig zu Musikern werden. Wie der Tanz hat die Wissenschaft einige implizite Ordnungs- und Organisationsformen, und auch diese ändern sich im Laufe der Zeit. Neuankömmlinge versuchen sich erst etwas zurechtzufinden, indem sie möglichst regelgetreu mithüpfen und die Erfahreneren imitieren. Später versuchen sie, auch ein bisschen den Ton anzugeben. Aber erst wenn sie richtig gut integriert sind im Gesamtkonzert, wird es ihnen gelingen, Einfluss auf die Musik zu nehmen, nach der im Moment alle tanzen.

Die komplementärmedizinische Forschung ist eine solche neue Mittänzerin auf einem vergleichsweise alten Parkett. Zwar hat es in der Medizin Tradition, dass sich neue Gebiete etablieren, zum Teil unter heftigen Gefechten. Meistens aber sind die verbindenden Elemente zwischen neuen Teilgebieten und alten Disziplinen stark genug. So haben sich etwa die neuen bildgebenden Verfahren nahtlos in die etablierten Disziplinen integriert. Andere Gebiete, wie die Palliativmedizin, haben es vielleicht etwas schwerer gehabt oder kämpfen immer noch um Gehör, aber die Gemeinsamkeit mit den Herkunftsdisziplinen ist immer noch größer als das Trennende.

Die komplementärmedizinische Forschung ist mit radikaleren Klängen angetreten. Sie hat nicht einen neuen, langsam innerhalb der Schulmeinung wachsenden Zweig zum Blühen gebracht und allmählich zu einem Baum gemacht, sondern gleichsam einen völlig anderen, neuartigen Baum, der bereits gepflanzt war, ins Zentrum des Interesses gestellt. So etwas ist immer schwieriger, als lediglich organisches Wachsen zu befördern. Man verlangt den anderen nicht nur ab, dass sie etwas neu Entstehendes als eigenständig wahrnehmen, sondern dass sie etwas ganz anderes, für das sie bislang gar keinen Blick hatten, ernst nehmen.

Es ist schon in normalen Familien nicht leicht, die langsam heranwachsenden Kinder in ihrer Selbständigkeit zu unterstützen und ihre oftmals anderen Meinungen, Ziele und Verhaltensweisen zu akzeptieren. Noch schwieriger ist es, Kinder, die schon mit relativ selbständiger Persönlichkeit zu einem späteren Zeitpunkt in die Familie kommen, zu integrieren.

\begin{tabular}{ll}
\hline KARGER & ( ) 2007 S. Karger GmbH, Freiburg \\
Fax +49761 4520714 & Accessible online at: \\
$\begin{array}{l}\text { E-mail Information@Karger.de } \\
\text { www.karger.com }\end{array}$ & www.karger.com/fok \\
&
\end{tabular}

Prof. Dr. Dr. Harald Walach

University College Northampton

School of Social Sciences \& Samueli Institute for Information Biology

European Office Boughton Green Rd, UK-Northampton NN2 7AL

Tel. +44 1604-892952, Fax -722067

E-mail harald.walach@northampton.ac.uk 
Eine derartige Herausforderung ist die komplementärmedizinische Forschung für das medizinische Establishment. Plötzlich tauchen Handlungs- und Denkweisen aus ganz anderen Kontexten auf und verlangen ihren Platz. Ob es sich dabei wie mit der traditionellen chinesischen Medizin, der Homöopathie, der klassischen Naturheilkunde oder der anthroposophischen Medizin um ganz eigenständige, mit der konventionellen Denkweise teilweise konkurrierende Therapiekonzepte handelt oder ob es einfach Teildisziplinen sind, die mit anderem Werkzeug operieren, wie die Neuraltherapie, die Phytotherapie oder die Chiropraktik, ist dabei unerheblich. Immer sind es Konzepte, Erfahrungen, ja Weltgestaltungen, die aus ihrem je eigenen Kontext kommend die Vielfalt menschlicher und medizinischer Erfahrung spiegeln und insofern - mit Recht - verlangen, in der einen oder anderen Form ernst genommen zu werden.

Die wissenschaftliche, vor allem medizinische Gemeinde hat in der Anfangsphase fast durchgängig mit Ablehnung reagiert. Diese erste Phase der komplementärmedizinischen Aktivität war demgemäß vor allem apologetischer Natur: Man versuchte, seine Daseinsberechtigung durch Rückgriff auf historische, grundlegende oder Konsumargumente zu belegen und durch braves Mitspielen den Mustertänzer zu geben, auf dass man zugelassen würde zum Ball der Mächtigen. Man versuchte, die Regeln mindestens so gut anzuwenden wie die anderen und mindestens so gute oder sogar bessere Studien durchzuführen als die konventionelle Konkurrenz. Diese erste Phase der Anpassung, wie ich sie nennen will, ist nun allmählich vorbei. Hat sie den gewünschten Erfolg gebracht? Ja und nein.

Ja, insofern als Strukturen entstanden sind. Arbeitsgruppen haben sich geformt und etabliert. Erste akademische Positionen gibt es. In Deutschland zwar noch weniger als anderswo, aber immerhin. Diverse Publikationsorgane und entsprechend viele Manuskripte haben einen ganz neuen Markt geschaffen. Wir dürfen stolz sein, mit der ForsCHENDEN KOMPLEMENTÄRMEDIZIN ein prägender Akteur auf diesem Markt zu sein. Auch Förderungsstrukturen sind entstanden, zwar nicht überall und durchgängig, aber immerhin. In den USA ist das National Center for Complementary and Alternative Medicine (NCCAM) zu einem nicht mehr wegzudenkenden Motor der Förderung geworden. Wenn wir Glück haben, wird sogar im nächsten Rahmenprogramm der EU komplementärmedizinische Forschung explizit ausgewiesen sein. In der Gesetzesvorlage ist sie das bereits; ob auch die Arbeitsdokumente entsprechend gefasst werden, wird sich in Kürze weisen. Die Chancen stehen jedenfalls gut. Viele Initiativen haben sich gebildet. EURICAM versucht, ein Netzwerk für Forscher auf europäischer Ebene zu schaffen (www.euricam.org). Einzelne Gruppierungen, etwa an Akupunktur oder Homöopathie interessierte Forscher, haben begonnen, sich zu formieren und ihre Interessen zu artikulieren. Vor einigen Jahren wurde die International Society for Complementary Medical Research (ISCMR; www.iscmr.org) gegründet mit dem Ziel, die internationale Forschergemeinde zusammenzuführen und ihr eine
Plattform und Austauschmöglichkeiten zu schaffen. Ich unterstütze als derzeitiger Vizepräsident und künftiger Präsident diese Ziele aktiv. Wir haben es geschafft, die bislang verstreuten und teilweise sehr lokalen Konferenzaktivitäten global zu strukturieren, so dass sich internationale und nationale Konferenzen künftig über die Kontinente verteilt abwechseln und eine wirklich internationale Vernetzung stattfinden wird. So wird dieses Jahr vom 11.-13. Mai in München eine große internationale Konferenz stattfinden, bei der die ISCMR als Mitorganisatorin auftritt. Die Abstracts dieser Konferenz werden in einem Supplementheft der ForsCHENDEN KOMPLEMENTÄRMEDIZIN veröffentlicht. Wir hoffen, künftig jährlich solche Treffen mit jeweils wechselnden lokalen Organisatoren durchführen zu können. 2008 werden wir uns in Australien treffen, danach vielleicht wieder in den USA, und dann zurück nach Europa. Daneben wird, soll und darf es andere Treffen geben, die andere Ziele verfolgen, anderes Publikum ansprechen. Denn das Ziel kann nicht sein, alles zu zentralisieren, sondern Vereinigung und Differenzierung zu ermöglichen. So muss und soll es regionale Verschiedenheiten geben, die die unterschiedlichen Situationen in verschiedenen Ländern oder Regionen widerspiegeln. Genauso müssen auch fachliche Spezialitäten ihren Platz haben. Innerhalb der ISCMR wird dies derzeit unter der Rubrik «regional chapters» und «special interest groups» verhandelt. So sind wir also wissenschaftssoziologisch gesehen an einem Punkt angelangt, an dem es einerseits notwendig wird, Kräfte zu bündeln und sich zusammenzuschließen, aber andererseits gleichzeitig zu differenzieren.

Die Differenzierung ist auch notwendig als Lehre aus den Misserfolgen, die sich beim Einhalten der Spielregeln bislang ergeben haben. So können alle Erfolge nicht darüber hinwegtäuschen, dass das Bravsein von den Lehrern und Meistern bislang nur unzureichend belohnt wurde. Gewiss, es wird immer diejenigen geben, die sagen: Ihr wart halt nicht brav genug, eure Studien waren eben nicht ausreichend gut, ausreichend vielfältig, ausreichend groß, ausreichend dies und ausreichend das. Ihr müsst euch erst noch mehr anstrengen, dann dürft ihr auch mitspielen. Und sicherlich: die ersten 10 Jahre Forschung auf dem Gebiet haben uns gerade mal gelehrt, wie man es nicht machen soll und wie es richtig geht.

Es haben sich aber auch profunde Probleme aufgetan, mit denen wir nicht gerechnet haben. Am Schweizerischen Programm Evaluation Komplementärmedizin (PEK) [1] konnte man plötzlich sehen: es geht gar nicht um Wissenschaftlichkeit, Daten oder Erkenntnis, sondern um Macht, Politik und Wirtschaftsinteressen [2]. An der Diskussion um die Integration der Akupunktur ins öffentliche Gesundheitssystem in Deutschland konnte man erkennen: unterschiedliche Akteure haben völlig verschiedene Interessen, sprechen verschiedene Sprachen und legen je andere Maßstäbe an. Die großen deutschen Akupunkturstudien [3-9] waren nicht deshalb möglich, weil die Öffentlichkeit ein Interesse an Aufklärung und Wissen hat, die Wissenschaftler ihrem öffentlichen Auftrag nachgekommen sind und die öffentliche Hand wie sich das eigent- 
lich gehören würde, diejenige Forschung förderte, an der ein breites öffentliches Interesse besteht. Nein, die Kassen nutzten das gesetzliche Instrument der Erprobungsverfahren, um die Nachfrage ihrer Klientel nach Akupunktur zu bedienen. Der Bundesausschuss «Ärzte und Krankenkassen», der festlegt, was bezahlt wird und was nicht, versuchte zunächst, eine große Anzahl von Akupunkturärzten von den Fressnäpfen der anderen fernzuhalten, damit der Gesamtkuchen nicht kleiner wird. Erkenntnisargumente - Wirksamkeit! Spezifische Wirksamkeit! - wurden zu Kampfrufen und zu Machtmitteln. Würde man die Ergebnisse etwa der großen Migräneprophylaxestudie ernst nehmen [10], müsste man eigentlich konventionelle Migräneprophylaxe aus der von der Allgemeinheit finanzierten Versorgung nehmen, da sie nicht wesentlich wirksamer war als das Akupunkturplacebo. Wird irgendjemand diese Schlussfolgerung ziehen? Vermutlich außer mir und einigen anderen Wildgewordenen niemand. Ich möchte nicht wissen, wie viele alternde Männer nach einer Viagra-Tour bei der Freundin oder im Bordell an einem Herzinfarkt sterben. Vermutlich mehr als die sieben Fälle von Leberversagen, die das Bundesinstitut für Arzneimittel und Medizinprodukte (BfArm) zur Rücknahme der Zulassung Kavahaltiger Präparate bewogen hat. Die Viagra-Herzinfarkte werden wohl nie als Nebenwirkung gewertet werden. Das Mitspielen nach den Regeln und die gewonnene Erkenntnis sind nur die halbe Miete, der Rest ist Macht, Politik und soziales Agieren.

Was heißt also «Verbinden und Differenzieren»? Wir müssen erkennen, dass das Mitspielen in der Wissenschaft keine Einwegkommunikation ist, nicht nur Hören auf die Musik, die gespielt wird, und sich entsprechend Bewegen. Es geht auch darum, diese Musik zu verändern. Die komplementärmedizinische Forschung kann hier wichtige Beiträge zur Differenzierung leisten. Das NCCAM und das NIH haben bereits einen wesentlichen Impuls aufgegriffen: Sie fördern prioritär Studien, die sich die Aufklärung von Selbstheilungsprozessen zum Ziel gesetzt haben. Früher haben wir alle abschätzig «Placeboeffekte» dazu gesagt, pfui!, nun heißen sie «nonspecific therapeutic effects», «therapeutic context effects», «effects of meaning and expectation» $[11,12]$. Plötzlich wird der Hintergrund und Abfall von Gestern zum Vordergrund und Gold von Morgen! Welch spannende Entwicklung! Und auf einmal gestalten wir die Tagesordnung der Forschung mit, setzen wir Schwerpunkte, die vor 10, ja vor 5 Jahren noch undenkbar waren (in Deutschland dauert natürlich alles wieder ein bisschen länger; hier wird die Deutsche Forschungsgemeinschaft (DFG) noch ihre 5 Jahre brauchen, bis die Wichtigkeit des Themas erkannt wird, aber ich würde sagen, es besteht Hoffnung). Nun gilt es also, begriffliche Differenzierungen, die sich aus der komplementärmedizinischen Forschung ergeben haben, in die konventionelle Agenda zu schreiben. Nun heißt es mit einem Mal, eine gemeinsame Sprache zu finden. Wenn wir uns jetzt mit der oftmals von uns ausgegrenzten «Mainstreamforschung», der «Schulmedizin», der «Orthodoxie» und wie die subtil abwertenden Begriffe alle lauten, verbinden, dann entdecken wir plötzlich: So verschieden sind unsere Probleme und Ansätze gar nicht. Wir sind nicht nur die Neulinge, die erst mal den Marschschritt einüben müssen. Nein, wir können die Musik subtil verändern, aus dem Marsch wird erst ein Ländler, dann ein Zwiefacher und ehe wir's erwarteten, ist ein Walzer draus geworden ...

Wissenschaft ist einfach ein spannender, ein historisch einmaliger und oft auch ein sehr witziger Prozess (wir merken es nicht immer: aber die Geschichte hat auch viel Humor). Mir scheint, ein Schlüssel, um diesen Prozess mitzubestimmen (und den Humor zu verstehen), ist das dialektische Denken und innerhalb dessen das vielleicht komplementäre Paar «Verbinden und Differenzieren». Verbinden und zusammenschließen: untereinander sowie mit den vermeintlich anderen und Feindlichen; gleichzeitig differenzieren und seine Eigenheit behaupten: untereinander, indem wir keinen Einheitsbrei kochen, aber auch nach außen, indem wir unsere Stärken und Eigenarten behalten. Damit können wir auch einen wichtigen Gegenpol setzen zu dem linear-spalterischen Denken, das die Welt in richtig und falsch, gut oder böse einteilt und dessen fatale Folgen wir allenthalben sehen.

\section{Literatur}

$>1$ Heusser P, Walach H, Wolf U (Hrsg): Kurzfassungen der HTA-Berichte des Schweizerischen PEK. Programm Evaluation Komplementärmedizin. Forsch Komplementärmed 2006;13:suppl 2.

2 Walach H: Economy chased medicine: Ökonomisierung der Gesundheit - Chance oder Bedrohung für die Komplementärmedizin? (Editorial). Forsch Komplementärmed Klass Naturheilkd 2005;12: 188-189.

3 Becker-Witt C, Brinkhaus B, Jena S, et al: Efficacy of acupuncture in patients with pain due to osteoarthritis of the knee - the Acupuncture Randomised Trials (ART) (Abstract). Focus Altern Complement Ther 2003;8:479-480.

$\checkmark 4$ Brinkhaus B, Becker-Witt C, Jena S, et al: Acupuncture randomized trials (ART) in patients with chronic low back pain and osteoarthritis of the knee - design and protocols. Forsch Komplementärmed Klass Naturheilkd 2003;10:185-191.
5 Brinkhaus B, Witt CM, Jena S, et al: Acupuncture in patients with chronic low back pain: a randomized controlled trial. Arch Intern Med 2006; 166:450-457.

6 Linde K, Streng A, Jürgens S, et al: Acupuncture for patients with migraine: A randomized controlled trial. J Am Med Association 2005;293: 2118-2125.

7 Melchart D, Linde K, Streng A, et al: Acupuncture randomized trials (ART) in patients with migraine or tension-type headache - design and protocols. Forsch Komplementärmed Klass Naturheilkd 2003; 10:179-184.

8 Melchart D, Streng A, Hoppe A, et al: Acupuncture in patients with tension-type headache: randomised controlled trial. Br Med J 2005;331: 376-382. $\checkmark$ Witt C, Brinkhaus B, Jena S, et al: Acupuncture in patients with osteoarthritis of the knee: a randomised trial. Lancet 2005;366:136-143.

10 Diener HC, Kronfeld K, Boewing G, et al: Efficacy of acupuncture for the prophylaxis of migraine: A multicentre randomised controlled clinical trial. Lancet Neurol 2006;5:310-316.

11 Walach H, Sadaghiani C: Plazebo und PlazeboEffekte: Eine Bestandsaufnahme. Psychother Psychosom Med Psychol 2002;52:332-342.

12 Walach H, Sadaghiani C, Dehm C, Bierman DJ: The therapeutic effect of clinical trials: understanding placebo response rates in clinical trials - a secondary analysis. BMC Med Res Methodol 2005;5: 26. 